This article was downloaded by: [TIB-Lizenzen - TIB Licence Affairs]

On: 18 August 2008

Access details: Access Details: [subscription number 777306420]

Publisher Taylor \& Francis

Informa Ltd Registered in England and Wales Registered Number: 1072954 Registered office: Mortimer House, 37-41 Mortimer Street, London W1T 3JH, UK

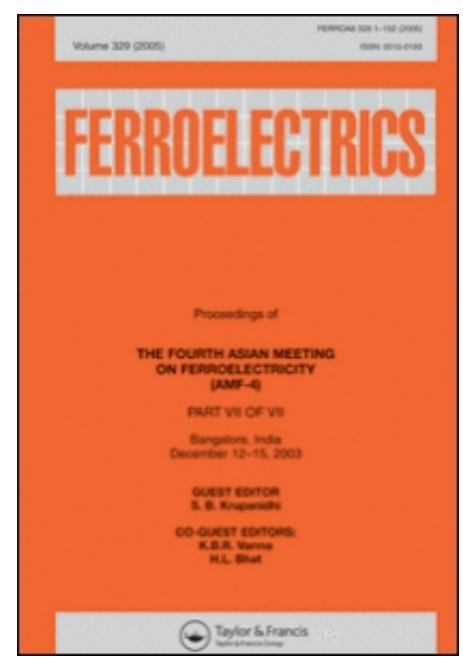

\title{
Ferroelectrics
}

Publication details, including instructions for authors and subscription information:

http://www.informaworld.com/smpp/title content=t713617887

\section{The electroporation hysteresis}

\section{Eberhard Neumann}

a Faculty of Chemistry, Department of Physical and Biophysical Chemistry, University of Bielefeld, Bielefeld, F.R. Germany

Online Publication Date: 01 October 1988

To cite this Article Neumann, Eberhard(1988)'The electroporation hysteresis',Ferroelectrics, 86:1,325 — 333

To link to this Article: DOI: $10.1080 / 00150198808227023$

URL: http://dx.doi.org/10.1080/00150198808227023

\section{PLEASE SCROLL DOWN FOR ARTICLE}

Full terms and conditions of use: http://www.informaworld.com/terms-and-conditions-of-access.pdf

This article may be used for research, teaching and private study purposes. Any substantial or systematic reproduction, re-distribution, re-selling, loan or sub-licensing, systematic supply or distribution in any form to anyone is expressly forbidden.

The publisher does not give any warranty express or implied or make any representation that the contents will be complete or accurate or up to date. The accuracy of any instructions, formulae and drug doses should be independently verified with primary sources. The publisher shall not be liable for any loss, actions, claims, proceedings, demand or costs or damages whatsoever or howsoever caused arising directly or indirectly in connection with or arising out of the use of this material. 
Ferroelectrics, 1988, Vol. 86, pp. 325-333

Reprints available directly from the publisher Photocopying permitted by license only
(C) 1988 Gordon and Breach Science Publishers S.A.

Printed in the United States of America

\title{
THE ELECTROPORATION HYSTERESIS
}

\author{
EBERHARD NEUMANN \\ Faculty of Chemistry, Department of Physical and Biophysical Chemistry, \\ University of Bielefeld, P.O. Box 8640, D-4800 Bielefeld 1, F.R. Germany
}

(Received 25 July 1988)

\begin{abstract}
Electroporation and electrofusion have developed to powerful methods in cell biology and gene technology. However, the molecular mechanisms of the electroporative gene transfer and of the electric membrane fusion are still not known. Electroporation renders the membrane transiently porous and at the same time fusiogenic. In the present digression electroporation is discussed in terms of structural rearrangements in the membrane. The onset of electric pore formation is viewed as a critical phenomenon. In order to cover the reversible as well as the irreversible processes of electroporation and electrofusion, the concept of the relaxation hysteresis of membrane electroporation is introduced.
\end{abstract}

\section{INTRODUCTION}

In physical chemistry and biophysics electric field methods have traditionally been applied to probe the ionic-electric properties and reactivities of molecules and molecular organizations such as biological membranes. ${ }^{1,2}$ Nowadays electric field pulse techniques also gain increasing importance in cellular and molecular biology, in gene technology and in medicine. In particular, the methods of electroporation ${ }^{3}$ and electrofusion ${ }^{4-7}$ have developed to powerful tools for cell manipulations (for reviews see references ${ }^{8-10}$ ) and for the physical chemical study of electrically induced structural rearrangements in membranes. ${ }^{2}$

Until now there is only indirect evidence that the applied electric pulses cause structural reorganizations in the cell membranes; for early reference see Sale and Hamilton, ${ }^{11}$ Neumann and Rosenheck, ${ }^{12}$ Lindner et al. ${ }^{13}$ Although there are a couple of model approaches toward theories for electropermeabilization and electrofusion ${ }^{9,10,11-12}$ it is fair to say that the detailed mechanisms of field-induced restructuring of membranes in electroporation and electrofusion processes are not known. In addition, in this new field there is still a need to classify the observations in terms of physical concepts and to estabilish an unequivocal terminology based on physical chemical principles. In the present account, electroporation and electrofusion are discussed in terms of field-induced structural rearrangements in the membranes. In particular, electroporation is viewed as a critical phenomenon and the concept of the relaxation hysteresis is introduced to elucidate the reversible and the irreversible aspects of electroporation and electrofusion.

\section{THRESHOLD AND STRENGTH-DURATION PARAMETERS}

There are several experimental parallels between electroporation and electrofusion. Both field effect phenomena show threshold behavior. For a given cell the 
numerical values of the threshold field strenghts $E_{c}$ are (almost) the same and depend inversely on the cell diameter. No doubt, both cell fusion and electroporative material exchange are clearly induced by the external field pulse. But, because of the longevity of the field-induced structural changes, the actual fusion events and the main part of the material exchange are, by and large, after-field effects. ${ }^{3,13,23-25}$

In summary the data suggest that it is one and the same primary field effect on the membrane structure: leading to cell fusion if cell membranes are brought into contact before or after pulsing, ${ }^{23}$ or causing DNA uptake if the DNA is adsorbed to the cell surface before (or after) pulsing.

\section{Threshold Parameters}

The threshold field strength $E_{c}$ for electroporation (electropermeabilization and electrofusion) is a kind of "point of no return". ${ }^{18}$ If the electric field $E\left(\geq E_{c}\right)$ is maintained, the electropores induced by the supercritical field increase in number and size ${ }^{14,15,21}$ until, at a supercritical number density and pore size, the membrane ruptures (dielectric breakdown). If electric pulses of short duration $\Delta t$ are applied, the field is already switched off before rupture can occur and the previous low-permeability state is apparently restored completely.

It is therefore pertinent to view membrane electroporation as a critical phenomenon, characterized by critical values for the extent $\xi_{c}$ of structural rearrangement, for the field strength $E_{c}$ and for the pulse duration $\Delta t_{c}$. In our structural model the primary requirement for the onset of electroporation is that the threshold $\xi_{c}$ has to be reached; see Figure 1. In this context the subcritical
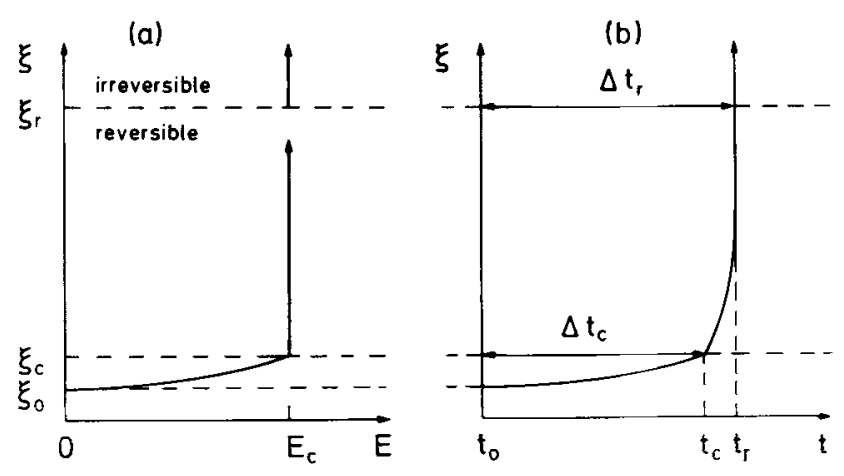

FIGURE 1 Electroporation viewed as a critical membrane phenomenon associated with critical values for the field strength $E_{c}$, the pulse duration $\Delta t_{c}$ and the degree of structural rearrangement $\xi_{c}$. (a) electroporation starts when the threshold $\xi_{c}$ is attained; the minimum field strength to reach $\xi_{c}$ is $E_{c}$. At $E \geq E_{c}$ the electroporation process is unidirectional, i.e. irreversible, until the rupture threshold $\xi_{r}$ is reached where the membrane breaks down irreversibly. If however, the field is switched off before $\xi_{r}$ is attained, the electroporated membrane reseals (reversible electroporation cycle). (b) The critical pulse duration $\Delta t_{c}$ to trigger the electroporation process at $\xi_{c}$ is the smaller the larger the field strength $E\left(>E_{c}\right)$. The reversible electroporation cycle requires that the pulse length $\Delta t$ is smaller than the rupture time $\Delta t_{r}$. The changes of $\xi$ from $\xi_{0}$ to $\xi_{c}$ represent reversible subcritical rearrangements such as, e.g., the increase of size and number of hydrophobic defect sites. 

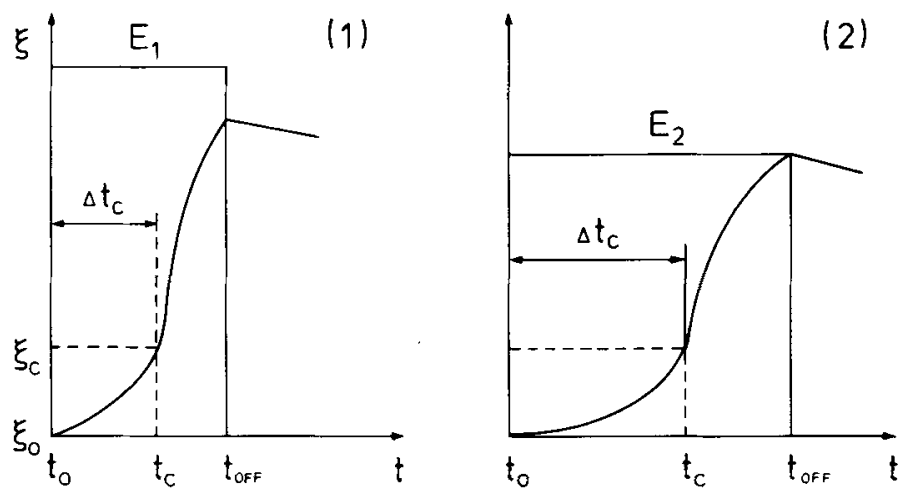

FIGURE 2 Strength/duration $\left(E / \Delta t_{c}\right)$ relationship for the onset of the electroporation process at $\xi=\xi_{c}$. The critical pulse length $\Delta t_{c}$ is the smaller the larger the field strength: $\Delta t_{c}\left(E_{1}\right)<\Delta t_{c}\left(E_{2}\right)$, if $E_{1}>E_{2}$.

changes from $\xi_{0}$ to $\xi_{c}$ represent reversible structural rearrangements such as e.g., the increase in number and size of hydrophobic defect sites and micropores in the bilayer. The minimum field strength to attain the critical value $\xi_{c}$ is the critical field $E_{c}$. Once the threshold $\xi_{c}$ is reached $\left(E \geq E_{c}\right)$ the actual electroporation starts and proceeds unidirectionally (no return) until the rupture threshold $\xi_{r}$ is attained where the membrane disintegrates. If the field is reduced below $E_{c}$ or switched off before $\xi_{r}$ is reached, the electropores or electrocracks ${ }^{22}$ reseal or anneal such that the original membrane state appears to be completely restored (reversible electroporation). ${ }^{18}$

\section{Strength-Duration Relationship}

Similar to other electric membrane phenomena such as nerve excitation, the onset of electroporation is associated with a strength-duration relationship. Since the threshold $\xi_{c}$ is atained faster at a higher field strength (Figure 1), the minimum pulse duration $\Delta t_{c}$ that is required for the onset of the electroporation process is the smaller the larger the applied external field (Figure 2).

If indeed the value $E_{c}$ decreases with the independently choosen pulse length $\Delta t{ }^{26}$ this feature may result from the stochastic nature of electropore formation. ${ }^{18,22}$ The larger $\Delta t$ the larger is the probability of nucleation of the electropores at a smaller field strength.

The organization of biological membranes is highly complex: uneven surface distribution and uneven membrane thickness; structural coupling to external matrix and to intracellular cytoskeletal elements. It is therefore not possible to exactly calculate the strength-duration parameter set $E_{c} / \Delta t_{c}$ from first principles.

The usual expression given for $E_{c}$ in terms of the cell radius $a$ and the critical transmembrane voltage $\left(V_{m, c}\right)$ is only an approximation. The approximation for the maximum value at the pole cap regions (where $\cos \delta=+1,-1$, respectively; see also Figure 3 ) is given by

$$
E_{c} \approx 2\left|V_{m, c}\right| / 3 a
$$



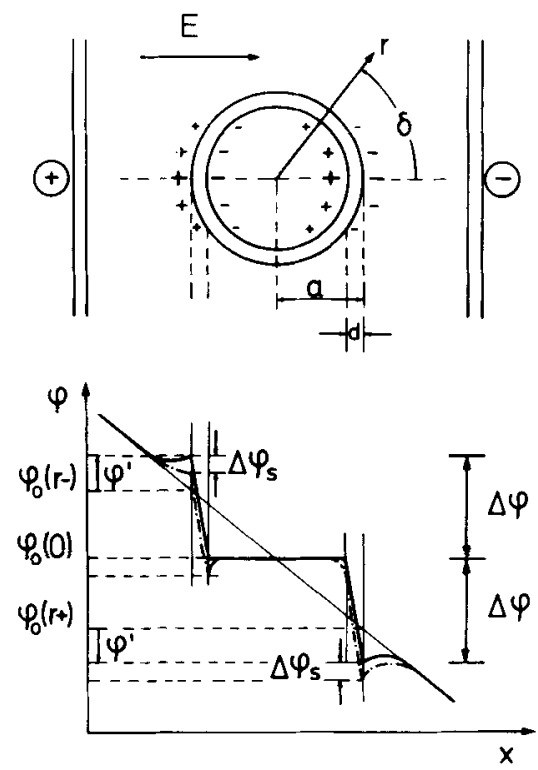

FIGURE 3 Interfacial polarization of a spherical nonconducting shell of thickness $d$ and outer radius $r=a$ in a constant external field $\mathbf{E}$. The stationary electric potentials are given in polar coordinates of the radius vector $\mathbf{r}$ and the angle $\delta$, such that the conducting interior of the cell has the constant reference potential $\varphi_{0}=0$ for $0 \leqq r \leqq(a-d)$. For $r>a, \varphi_{0}=-\mathbf{E} \cdot \mathbf{r}=-E \cdot r \cos \delta$. The total potential $\varphi(r)$, relative to $\varphi_{0}(0)=0$, is given by $\varphi(r, \delta)=\varphi_{0}(r, \delta)+\varphi^{\prime}(r, \delta)$, where $\varphi^{\prime}(r, \delta)$ is the contribution of the interfacial polarization. The $\Delta \varphi$ terms are the interfacially induced, crossmembrane potential differences in the absence of fixed ionic groups and adsorbed ions, $\left(\Delta \varphi_{s}=0\right)$. The dash/point line models schematically the potential profile in the presence of fixed surface charges (here negative).

Equation (1) is practically very useful, even for the estimate of the threshold of the initial field strength of exponentially decaying pulses (CD-pulses); $V_{m, c} \approx 0.5$ to $1 \mathrm{~V}$ for short duration pulses $(\Delta t \approx 10 \mu \mathrm{s})$ and $V_{m, c} \approx 0.2-0.5 \mathrm{~V}$ for longer pulse duration $(\Delta t \geq 0.1 \mathrm{~ms})$.

\section{FIELD AMPLIFICATION BY INTERFACIAL POLARIZATION}

The magnitude of the applied field strength and the dependence of the threshold $E_{c}$ on the cell radius indicate that the field effect on the membrane structure is indirect. The data suggest that interfacial polarization precedes the structural transitions.

The actual membrane field affecting the lipids and proteins is strongly amplified by the interfacial polarization ${ }^{27,24}$ The time constant $\left(\tau_{p}\right)$ of the build-up of the interfacial polarization $\Delta \varphi$ is dependent on $a$ and the conductivities $(\lambda)$ of the cell interior, the cell membrane and of the external medium. ${ }^{27}$ In brief, the electroporation and electrofusion data indicate the sequence of events: ${ }^{28}$

$$
E \rightarrow \Delta \varphi \rightarrow \Delta \xi
$$




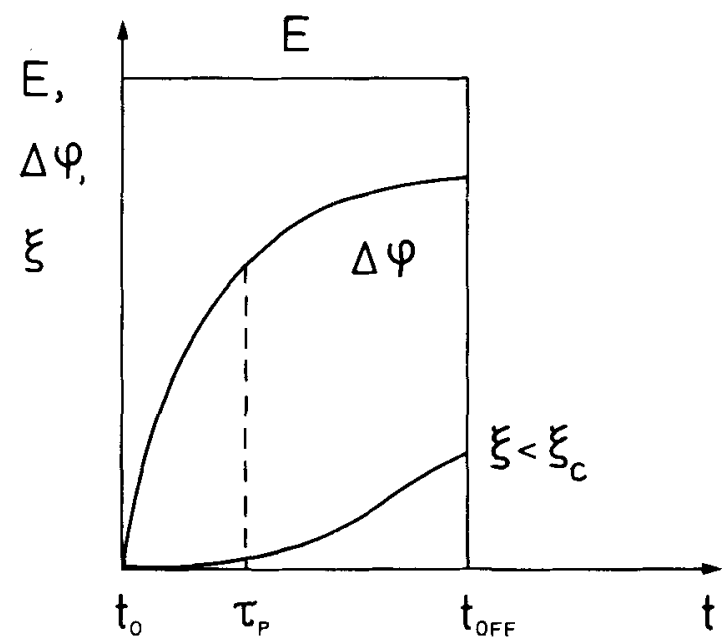

FIGURE 4 Amplified field effect. The external field pulse $(E, \Delta t)$ causes the interfacial potential difference $\Delta \varphi$. The electric field, equivalent to $\Delta \varphi$, in turn induces structural rearrangements $\xi(t)$ in the membrane. Thus $\xi(t)$ is delayed with respect to the application of $E$ at $t_{0}$. The time course $\Delta \varphi(t)$ is represented as a simple exponential process (time constant $\tau_{p}$ ). If the pulse duration at $E$ is $\Delta t<\Delta t_{c}$, the time course $\xi(t)<\xi_{c}$ models a subcritical change.

where $E$ causes the change $\Delta \varphi$, and $\Delta \varphi$ in turn causes the change in the extent $\Delta \xi$ of membrane rearrangements. In this sense the $\xi(t)$ function is delayed with respect to the application of the field pulse (Figure 4).

It is well known that all cell membranes have a natural electric potential difference, $\Delta \varphi_{m}$; typically, $\Delta \varphi_{m}=-70$ to $-100 \mathrm{mV}$ relative to the outside potential (zero).

The stationary value of the actual transmembrane voltage $V_{m}$, relative to the direction of the (constant) external field vector $\mathbf{E}$, results from contributions from the (diffusion) potential $\Delta \varphi_{m}$, from asymmetric surface charges $\left(\Delta \varphi_{s}\right)$ and the interfacial polarization. The voltage drop $V_{m}$ in the direction of $E$ is given by: ${ }^{28}$

$$
V_{m} \approx-\Delta \varphi_{m} \frac{|\cos \delta|}{\cos \delta}-\frac{3}{2} f(\lambda) E a|\cos \delta|
$$

where $E$ is the amount of $\mathbf{E}$ and the conductivity factor $f(\lambda) \leq 1$ may be approximated by $f(\lambda)=1$ for a nonconducting membrane. Equation (3) correctly covers the signs and the angular position dependence of $V_{m}$ relative to $\mathbf{E}$. At the pole caps in the $\mathbf{E}$ direction, $|\cos \delta|=1$, yielding the maximum values of $V_{m}$.

An average value for the stationary transmembrane electric field strength $E_{m}$ relative to the external field vector may be estimated from:

$$
E_{m} \approx-V_{m} / d
$$

where $d$ is the membrane thickness. 


\section{THE RELAXATION HYSTERESIS OF ELECTROPORATION}

Without doubt, the electroporation and electrofusion data indicate that on the one hand reversible primary processes and on the other hand irreversible secondary events are involved; see Table I.

Ionic-dielectric polarization of the membrane/solution interfaces and structural rearrangements in the membrane are essentially reversible processes. Material exchange and fusion processes are passive, unidirectionally occurring relaxation phenomena of irreversible nature. Furtheron, the data indicate that direct external field effects on the membrane structure are of minor extent. Rather, interfacial polarization at first leads to the strong (amplified) transmembrane field which in turn induces the major structural rearrangements. ${ }^{28}$

Obviously, a transient permeability increase ${ }^{12}$ indicates transient membrane

TABLE I

Fundamental processes of the electroporation hysteresis of membranes

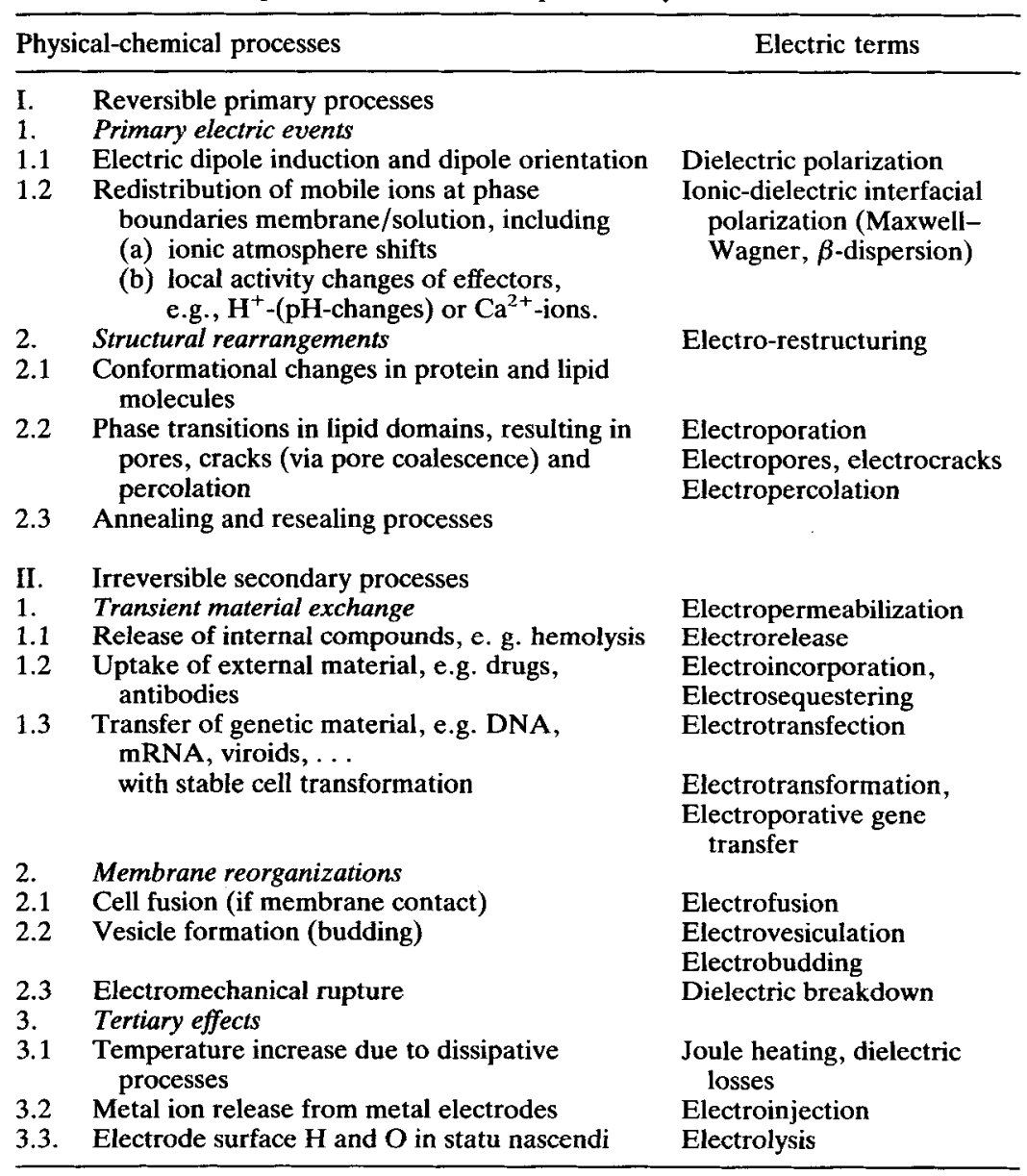


"openings": pores or cracks which reseal after pulsing. When the cycle of permeability increase and decrease is modelled on the level of electropores, ${ }^{14,18,22}$ the formation-resealing cycle of a pore is represented as a cyclic local change of the membrane structure. In addition, local deformations of the pore edges may lead to crater-like pore structures. ${ }^{28}$

\section{Metastable States and Undirectional Transitions}

As outlined previously, the apparent dependence of the threshold field strength $E_{c}$ on the pulse length ${ }^{26}$ indicates a membrane specific critical threshold $\xi_{c}$ at which the electroporation process is triggered. Once initiated, the process is unidirectional, i.e., irreversibly running at constant $E\left(>E_{c}\right)$. Therefore, the membrane state just before the onset of the electroporation process must be metastable. The minor structural rearrangements before electroporation $\left(\xi_{0}<\right.$ $\xi<\xi_{c}$ ) are apparently reversible but occur on a metastable level. ${ }^{29}$

At short pulse durations $\left(\Delta t<\Delta t_{r}\right)$ such that the rupture threshold $\xi_{r}$ was not reached, the electroporated (and fusiogenic) membrane is again metastable with respect to less porous structures. The pore resealing process (at $0<E<E_{c}$ ) is also unidirectional, i.e. irreversibly running until intact bilayer structures are restored.

In this sense membrane electroporation represents a cycle of structural rearrangements (Figure 5), where the intermediate states of the annealing process are probably different from those of the electroporation process in the presence of the external field.

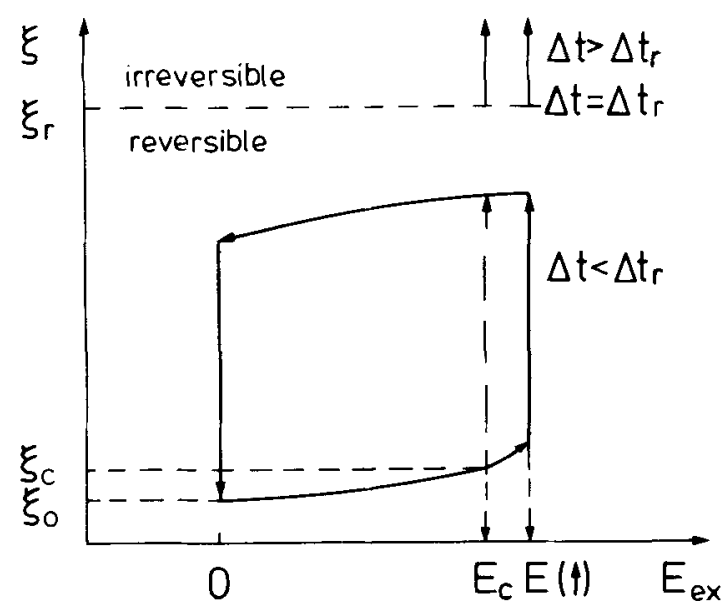

FIGURE 5 The relaxation hysteresis of the membrane electroporation. The cyclic change in the extent of structural rearrangements $\xi$ (at $E>E_{c}$ and $\Delta t(E)<\Delta t_{r}$ ) are displayed as a function of the external field $\left(E_{\mathrm{ex}}\right)$. The cycle comprises reversible as well as irreversible elements. The subcritical changes of $\xi$ between $\xi_{0}$ and $\xi_{C}\left(E<E_{c}\right)$ are reversible. At the supercritical field strength $E$ ( $\left.\uparrow\right)$ $\left(>E_{c}\right)$ the structural rearrangements associated with the electroporation process are unidirectional, i.e. irreversible. If the field duration is larger than the rupture threshold $\left(\Delta t_{r}\right)$, the membrane ruptures. If however the field pulse is switched off at $\Delta t<\Delta t_{r}$, we remain in the reversible electroporation domain. The (slow) return at $E=0$ from the upper branch to $\xi=\xi_{0}$ is unidirectional; the resealing or annealing processes are irreversibly occurring relaxations to the initial state $\xi_{0}$. 
The physical conception that comprises both (reversible) metastable states and (irreversible) unidirectional transitions in a cyclic manner is called hysteresis. ${ }^{29}$ Therefore the electroporation/resealing cycle may be analyzed and understood in terms of a structural relaxation hysteresis.

\section{Energetics of the Electroporation Hysteresis}

The energetics of the field-induced structural rearrangements in the electroporation/resealing cycle can be thermodynamically treated independent of any special mechanism.

The characteristic reaction free enthalpy $\Delta_{r} G(E)$ for the transition from the intact bilayer state to pore configurations is given by

$$
\Delta_{r} G(E)=\Delta_{r} G-\int_{0}^{E_{m}} \Delta_{r} M d E_{m}
$$

where $E_{m}$ is given by Equations (3) and (4) and $\Delta_{r} G$ is the reaction free enthalpy in the absence of $E .^{30,2}$ Obviously, pore formation is energetically unfavorable at $E=0$; hence $\Delta_{r} G>0$.

The reaction dipole moment $\Delta_{r} M$ is related to the difference between the moments $M_{w}$ of the water filled pore and $M_{m}$ of the bilayer having the same size as the aqueous pore.

Applying the dielectric continuum model the polarization moments (relative to vacuum) are given by

$$
M=\epsilon_{0}(\epsilon-1) v E_{m}
$$

where $\epsilon_{0}$ is the dielectric permittivity of the vacuum, $\epsilon$ the dielectric constant of the medium and $v$ is the volume of the electroporated membrane.

The polarization of the water $\left(\epsilon_{w} \approx 80\right)$ near the pore edge in the pore wall ${ }^{18}$ is energetically more favorable (by a factor of about 40) than the polarization of an equal volume of lipid bilayer $\left(\epsilon_{m} \approx 2\right) .{ }^{14}$ With $\Delta_{r} M=N_{A}\left(M_{w}-M_{m}\right)$, where $N_{A}$ is the Avogadro constant, we can specify

$$
\Delta_{r} M=N_{A} \epsilon_{0}\left(\epsilon_{w}-\epsilon_{m}\right) v(\delta) E_{m}
$$

If $d \ll a$, the electroporated membrane volume $v(\delta)$ is given by $v(\delta) \approx$ $4 \pi a^{2} d(1-|\cos \delta|)$ where the fraction of spherical membrane shell affected by a supercritical field is $(1-|\cos \delta|)=1-E_{c} / E$ (Figure 3).

It is seen from Equation (7) that, because of $\epsilon_{w}>\epsilon_{m}$, we obtain $\Delta_{r} M>0$. Therefore, the unidirectional electroporation process is associated with $\Delta_{r} G(E)<0$; obviously because $\int \Delta_{r} M d E_{m}>\Delta_{r} G$; see Equation (5). On the same line, the initial driving force for the unidirectional pore resealing process is given by $\Delta_{r} G_{\text {res. }}=-\left|\Delta_{r} G(E)\right|$.

In the case of unidirectional transitions, starting from metastable states, the rate equation for the extent $\xi_{i}$ of the $i$-th mode of the structural rearrangements,

$$
d \xi_{i} / d t=k_{i j} \xi_{i}-k_{i j}^{\prime}\left(1-\xi_{i}\right)
$$

is reduced to the simple form

$$
d \xi_{i} / d t=k_{i j} \xi_{i}
$$

Because of the irreversibility the reverse process can be neglected. The general 
rate coefficient $k_{i j}$ represents a combination of the rate constants $k_{j}$ of all elementary steps $j$ contributing to the mode $i$.

Finally, the field dependence of the rate constant $k_{j}$ is described by

$$
k_{j}(E)=k_{j}(0) e^{\int \Delta_{r} M_{j}^{*} d E / R T}
$$

where $\Delta_{r} M_{j}^{*}$ is the transition dipole moment of the activated state, $R$ the gas constant, $T$ the absolute temperature and $k_{j}(0)$ the value at $E=0$.

The general remarks and the explicit expressions given in this programmatic study may provide a general framework for the thermodynamic and kinetic analysis of the electroporation and electrofusion processes in terms of a hysteresis formalism.

\section{ACKNOWLEDGEMENTS}

The technical help by Mrs. A. Tiemann and Mrs. M. Pohlmann in the preparation of the figures and the typing of the manuscript is gratefully acknowledged. I thank the Deutsche Forschungsgemeinschaft for the grant DFG $\mathrm{Ne} 227 / 4$.

\section{REFERENCES}

1. M. Eigen and L. DeMaeyer, Techniques of Organic Chemistry, (John Wiley, New York, 1963) 8(2) pp. 895-1054.

2. E. Neumann, Prog. Biophys. molec. Biol., 47, 197-231 (1986).

3. E. Neumann, M. Schaefer-Ridder, Y. Wang and H. P. Hofschneider, EMBO J., 1, 841-845 (1982).

4. M. Senda, J. Takeda, A. Shunnosuke and T. Nakamura, Plant \& Cell Physiol., 20, 1441-1443 (1979).

5. E. Neumann, G. Gerisch and K. Opatz, Naturwissensch., 67, 414-415 (1980).

6. U. Zimmermann and P. Scheurich, Planta, 151, 26-32 (1981).

7. H. Weber, W. Förster, H.-E. Jacob and H. Berg, Z. Allgem. Mikrobiol., 21, 555-562 (1981).

8. H. Berg, studia biophysica, 119, 17-29 (1987).

9. A. E. Sowers, (ed.), Cell fusion, (Plenum Press, New York, 1987) 540 p.

10. U. Zimmermann, Rev. Physiol. Biochem. Pharmacol., 105, 175-255 (1986).

11. A. J. H. Sale and W. A. Hamilton, Biochim. Biophys. Acta, 163, 37-43 (1968).

12. E. Neumann and K. Rosenheck, J. Membr. Biol. 14, 194-196 (1973).

13. P. Lindner, E. Neumann and K. Rosenheck, J. Membr. Biol. 32, 231-254 (1977).

14. I. G. Abidor, V. B. Arakelyan, L. V. Chernomordik, Y. A. Chizmadzhev, V. F. Patushenko and M. R. Tarasevich, Bioelectrochem. Bioenerg., 6, 37-52 (1979).

15. R. Benz, F. Beckers and U. Zimmermann, J. Membr. Biol., 48, 181-204 (1979).

16. J. Teissié and T. Y. Tsong, Biochem., 20, 1548-1554 (1981).

17. D. S. Dimitrov and R. K. Jain, Biochim. Biophys. Acta, 779, 437-468 (1984).

18. I. P. Sugar and E. Neumann, Biophys. Chem., 19, 211-225 (1984).

19. J. C. Weaver, K. T. Powell, R. A. Mintzer, H. Ling, and S. R. Sloan, Bioelectrochem. Bioenerg., 12, 393-412 (1984).

20. K. T. Powell, E. G. Derrick and J. C. Weaver, Bioelectrochem. Bioenerg. 15, 243-255 (1986).

21. L. V. Chernomordik, S. I. Sukharev, S. V. Popov, V. F. Pastushenko, A. V. Sokirko, I. G. Abidor and Y. A. Chizmadzhev, Biochim. Biophys. Acta, 902, 360-373 (1987).

22. I. P. Sugar, W. Förster and E. Neumann, Biophy. Chem., 26, 321-335 (1987).

23. A. E. Sowers, J. Cell Biol., 99, 1989-1996 (1984).

24. E. Neumann, Bioelectrochem. Bioenerg., 16, 565-567 (1986).

25. E. Neumann, ZiF Proc., University of Bielefeld Press, pp. 6-14 (1986).

26. U. Zimmermann, P. Scheurich, G. Pilwat and R. Benz, Angewandte Chemie, 93, 332-351 (1981).

27. H. P. Schwan, Adv. Biol. Med. Physics, Vol. V. (Academic Press, New York, 1957) pp. 147-209.

28. E. Neumann, Electroporation and electrofusion in cell biology, (Plenum Press, New York, 1988) in press.

29. E. Neumann, Angew. Chem. internat. Edit., 12, 356-369 (1973).

30. G. Schwarz, J. Phys. Chem., 71, 4021-4030 (1967). 